The Impact of Large-Scale Surveys on Pulsating Star Research

ASP Conference Series, Vol. 203, 2000

L. Szabados \& D. W. Kurtz, eds.

\title{
New $\gamma$ Doradus Stars from the Hipparcos Mission and Geneva Photometry
}

\author{
Laurent Eyer, Conny Aerts ${ }^{1}$ \\ Instituut voor Sterrenkunde, Katholieke Universiteit Leuven, \\ Celestignenlaan 200 B, B-3001 Leuven, België
}

\begin{abstract}
A search for new $\gamma$ Dor stars was undertaken using the Hipparcos periodic variable star catalogue and the Geneva photometric database, leading to a list of 40 new candidates. We started a monitoring of the candidates which suited the observational window with the CORALIE spectrograph at the Swiss Euler Telescope for establishing a robust list of new $\gamma$ Dor stars and studying line profile variations. We present here our long-term program.
\end{abstract}

\section{Introduction}

The $\gamma$ Dor stars have amplitude variations up to $0.1 \mathrm{mag}$ in Johnson $V$ and periods ranging from 0.4 to $3 \mathrm{~d}$ (Kaye et al. 2000). We searched in two databases for finding new members of this class of variable stars. The first is the Hipparcos main mission photometric database. It contains a mean of 110 measurements for 118204 stars brighter than 12.4 and is magnitude complete up to 7.3-7.9 depending on the galactic latitude $b$. As the sampling is ruled by the scanning law of the satellite, it is not affected by the aliasing around $1 \mathrm{~d}^{-1}$, which might be a problem for detecting $\gamma$ Dor stars.

The second scanned database is the Geneva photometric catalogue (Burki \& Kienzle 2000), it counts 48000 stars and 345000 measurements in a sevencolour system. The content of the Geneva catalogue is the combination of more than 200 scientific programmes, including the Bright Star Catalogue south of $\delta<+20$.

\section{Hipparcos Main Mission and Geneva Photometry}

Thousands of new variable stars were discovered by the Hipparcos satellite. During the analysis of the Hipparcos photometry, stars from the Periodic Catalogue having accurate parallaxes and colours were plotted in the HR diagram (Eyer 1998). A clump of stars just at the cool lower edge of the $\delta$ Scuti instability strip was present and gave rise to a list of 15 candidates (excluding redundant cases from the other studies). A clump is also present when plotting the variable stars of the Hipparcos Unsolved Catalogue.

${ }^{1}$ Postdoctoral Fellow, Fund for Scientific Research, Flanders 
A systematic search for new $\gamma$ Dor stars was undertaken in the Hipparcos periodic variable star catalogue also using Geneva photometry and performing a multivariate discriminant analysis. This study led to a list of 14 new $\gamma$ Dor stars (Aerts, Eyer, \& Kestens 1998). This method is strict, since information on amplitude, period, physical parameters and multiperiodic behaviour were taken into account.

\section{The Search in Geneva Photometry}

Finally, the Geneva photometric database was scanned to find $F$ dwarf stars with high standard deviations. Eleven candidates were then measured with the $70-\mathrm{cm}$ Swiss telescope, resulting in about 1000 photometric measurements, which are under study (Eyer \& Aerts, in preparation). It turns out that about half of the suspected stars might have constant brightness.

\section{Spectroscopic Measurements}

In order to confirm the pulsational character of these stars, new spectra have been taken with the CORALIE spectrograph on the 1.2-m Swiss telescope at ESO-La Silla. The photometry and spectroscopy are necessary steps since we want to establish a robust list of new $\gamma$ Dor stars. Up to now 22 stars have been measured; the strategy consists of taking at least five spectra of each candidate. Among the stars, some are binaries, some are fast rotators and some show clear line-profile variations (cf. Fig. 1). Some stars are too faint for the telescope size, thus correlation techniques are used to lower the noise level. The next step is to accumulate photometry and spectra for promising candidates in order to perform mode identification.

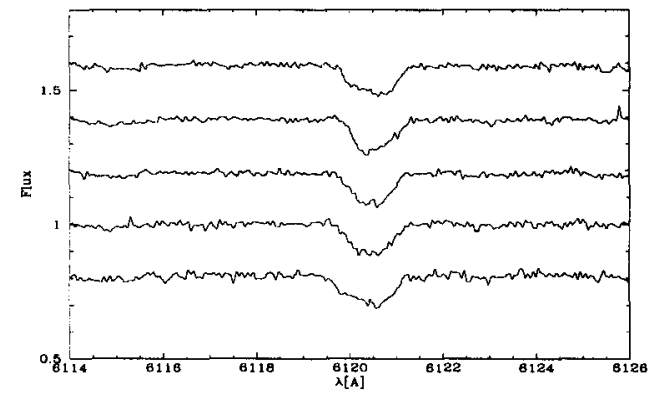

Figure 1. Line profile variations of the star HD 14940.

\section{References}

Aerts, C., Eyer, L., \& Kestens, E. 1998, A\&A, 337, 790

Burki, G. \& Kienzle, F. 2000, in these proceedings, p. 66

Eyer, L. 1998, PhD Thesis, Geneva University

Kaye, A., Handler, G., Krisciunas, K., Poretti, E., \& Zerbi F. M. 2000, in these proceedings, p. 426 